\title{
Some Extremal Results on Circles Containing Points
}

\author{
Ryan Hayward,' David Rappaport, ${ }^{2}$ and Rephael Wenger ${ }^{3}$ \\ 'Department of Computer Science, Rutgers University, Hill Center, \\ Busch Campus, New Brunswick, NJ 08903, USA \\ ${ }^{2}$ Computing and Information Science, Queen's University, Kingston, \\ Ontario K7L-3N6, Canada \\ ${ }^{3}$ McGill University, 805 Sherbrooke Street West, Montreal, \\ Quebec H3A-2K6, Canada
}

\begin{abstract}
We define $\Pi(n)$ to be the largest number such that for every set $P$ of $n$ points in the plane, there exist two points $x, y \in P$, where every circle containing $x$ and $y$ contains $\Pi(n)$ points of $P$. We establish lower and upper bounds for $\Pi(n)$ and show that $\{n / 27\}+2 \leq \Pi(n) \leq\{n / 4\rceil+1$. We define $\bar{\Pi}(n)$ for the special case where the $n$ points are restricted to be the vertices of a convex polygon. We show that $\vec{\Pi}(n)=\lceil n / 3\rceil+1$.
\end{abstract}

\section{Introduction}

Let $P$ be a set of $n$ points in the plane. A circle contains point $x$ if $x$ lies in the interior or on the boundary of the circle. For any two points $x$ and $y$ in a set $P$ of $n$ points in the plane, let $C(P, x, y)$ be the minimum number of points contained by any circle containing $x$ and $y$. Define $\pi(P)=\max \{C(P, x, y)\}$, over all pairs of points $x, y$ in $P$. A set $K$ of $n$ points in the plane will be called convex if the points form the vertices of a convex polygon. Define $\Pi(n)=\min \{\pi(P)\}$, over all sets $P$ of $n$ points in the plane, and define $\bar{\Pi}(n)=\min \{\pi(K)\}$, over all convex sets $K$ of $n$ points in the plane. Neumann-Lara and Urrutia [2] showed that

$$
\left\lceil\frac{n-2}{60}\right\rceil \leq \Pi(n) \text { and that }\left\lceil\frac{n-2}{4}\right\rceil \leq \bar{\Pi}(n) \text {. }
$$

In this paper we improve on their results by showing that

$$
\left\lfloor\frac{n}{27}\right\rfloor+2 \leq \Pi(n) \leq\left\lceil\frac{n}{4}\right\rceil+1 \text { and that } \bar{\Pi}(n)=\left\lceil\frac{n}{3}\right\rceil+1 .
$$




\section{The Convex Case}

In this section we prove:

Theorem 1. $\bar{\Pi}(n) \geq\lceil n / 3\rceil+1$.

Schmerl [3] provided a similar proof for this lower bound on $\bar{\Pi}(n)$. We borrowed some of his ideas to simplify our own presentation. We first state two lemmas which will be useful in bounding $C(P, x, y)$ in both the general and the convex case. We use the notation $(x y)$ to refer to the line segment from $x$ to $y$. We say that a closed region $R$ contains a point $x$ if $x$ lies on the interior or boundary of $R$.

Lemma 1. Given $P$, a set of $n$ points in the plane, $x, y \in P$, and a circle $\phi$ through $x$ and $y$, the line segment $(x y)$ divides circle $\phi$ into two closed regions $R_{1}$ and $R_{2}$. If $R_{1}$ and $R_{2}$ each contain $k$ points of $P$, then $C(P, x, y) \geq k$.

We leave the proof of Lemma 1 to the reader.

A spanning circle of $P$ is a circle containing all the points in $P$. A spanning circle $\phi$ of $P$ through at least three points $x, y, z$ of $P$ can always be found. These three points form a triangle, $\triangle x y z$, which divides circle $\phi$ into three closed regions bordered by arcs of $\phi$ and the triangle. We call these three closed regions, arc regions.

Lemma 2. Given a set, $P$, of $n$ points in the plane and an integer $t$, either:

(a) there exist two points $x, y \in P$ such that $C(P, x, y) \geq\lceil t / 3\rceil+2$, or

(b) there exists a triangle, $\triangle x y z$, containing $n-t+1$ points of $P$.

Proof. Let $P$ be any collection of $n$ points in the plane. For any three points, $x, y, z \in P$, with spanning circle $\phi$ through $x, y, z$, let $f(\triangle x y z)$ be the maximum number of points contained in each of the three arc regions created by $\triangle x y z$ and $\phi$. Choose three points $x, y, z \in P$, with spanning circle $\phi$, which minimizes $f(\triangle x y z)$. We claim that $x, y, z$ satisfy either condition (a) or condition (b).

Assume that $\triangle x y z$ does not contain $n-t+1$ points of $P$. Label the three arc regions bounded by $\triangle x y z$ and $\phi, A, B$, and $C$ (see Fig. 1). There must be at

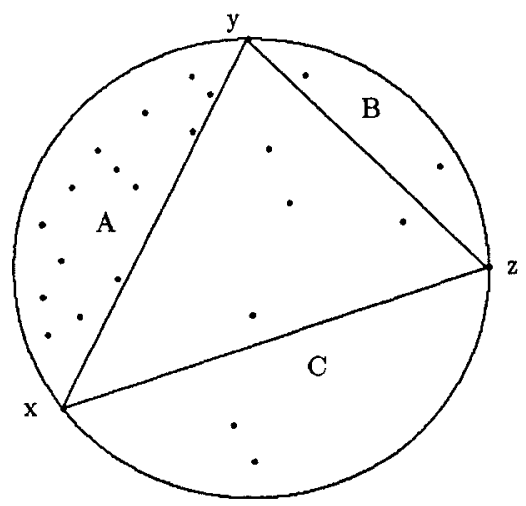

Fig. 1. Set of points divided by $\triangle x y z$. 


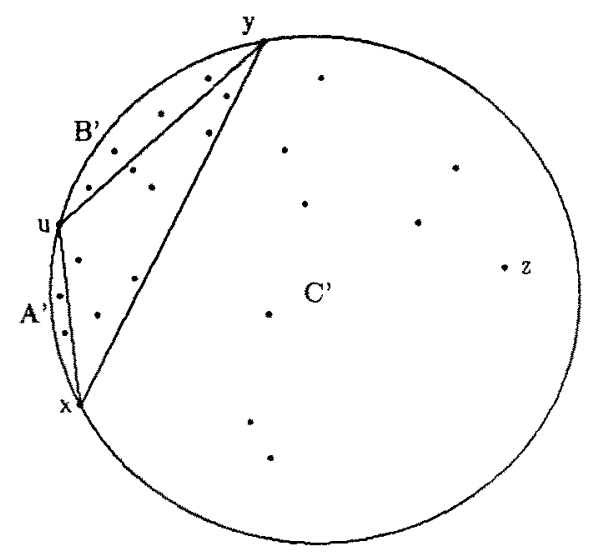

Fig. 2. New division by $\Delta x u y$.

least $t+3$ points in $A, B$, and $C$. (Note that points $x, y, z$ lie both in $\triangle x y z$ and in $A \cup B \cup C$.) Some region, $A, B$, or $C$, must contain at least $\lceil t / 3\rceil+2$ points. Without loss of generality, assume $A$, bordered by $(x y)$, does.

If $B \cup C \cup \triangle x y z$ contain fewer than $\lceil t / 3\rceil+2$ points, then choose point $u \in$ $P-\{x, y, z\}$ in region $A$ such that the circle $\phi^{\prime}$ through $x, y, u$ is a spanning circle of $P$. (We can find such a point $u$ by minimizing angle $x u y$ over all points other than $x$ and $y$ in region $A$.) $\Delta x u y$ divides circle $\phi^{\prime}$ into three new regions, $A^{\prime}, B^{\prime}, C^{\prime}$ (see Fig. 2). $C^{\prime}$ contains fewer than $\{t / 3\rceil+2$ points since it is composed of regions $B, C$, and $\triangle x y z . A^{\prime}$ and $B^{\prime}$ each contain fewer points than $A$. Thus, $f(\triangle x u y)<f(\triangle x y z)$, and $f(\triangle x y z)$ is not minimal, contrary to our assumption. Therefore, $B \cup C \cup \triangle x y z$ must contain at least $\lceil t / 3\rceil+2$ points. By Lemma 1 $C(P, x, y) \geq\lceil t / 3\rceil+2$.

For any set of convex points, $K$, any triangle, $\triangle x y z$, contains exactly three points. Setting $t$ equal to $n-3$ in Lemma 2, we conclude that there exists an $x, y \in K$, such that $C(K, x, y) \geq\lceil n / 3\rceil+1$. This proves Theorem 1 .

\section{The General Case}

We now prove:

Theorem 2. $\Pi(n) \geq\lfloor n / 27\rfloor+2$.

It suffices to prove that for $n \equiv 0 \bmod 27$ and any set, $P$, of $n$ points in the plane, there exist two points $x, y \in P$ such that $C(P, x, y) \geq n / 27+2$. For $n \equiv i \bmod 27$ we can delete $i$ points to form a set $P^{\prime}$ of $n-i$ points and find $x$ and $y$ such that $C\left(P^{\prime}, x, y\right) \geq(n-i) / 27+2$. It follows that $C(P, x, y) \geq\lfloor n / 27\rfloor+2$.

Neumann-Lara and Urrutia [2] presented the following lemma which relates the intersection of line segments to a property of circle containment. 
Lemma 3. If line segments $(x y)$ and ( $u v)$ intersect, then either every circle containing $x$ and $y$ contains $u$ or $v$, or every circle containing $u$ and $v$ contains $x$ or $y$.

One possible proof follows from Lemma 1 and is left to the reader. We need a fourth lemma concerning matchings which follows directly from many wellknown results in graph theory.

Lemma 4. Given a bipartite graph of $m$ edges and maximum vertex degree $k$, there exists a matching using $\lceil\mathrm{m} / \mathrm{k}\rceil$ of the edges.

Proof. In a bipartite graph, the number of edges in a maximum matching is equal to the number of vertices in a minimum vertex covering [1]. Since each vertex has degree $k$, at least $\lceil m / k\rceil$ vertices are needed to cover $m$ edges. Thus the minimum vertex covering has at least $\lceil\mathrm{m} / k\rceil$ vertices, and there is a matching using at least $\lceil m / k\rceil$ edges.

For any collection of $n$ points, $P$, where $n \equiv 0 \bmod 27$, we show how to find points $x, y \in P$ such that $C(P, x, y) \geq n / 27+2$. First, find a line splitting $P$ into two sets of $n / 3$ points and $2 n / 3$ points, respectively. Label the set of $n / 3$ points $S_{1}$.

Let $P^{\prime}$ be the set of $2 n / 3$ points which are not in $S_{1}$. Applying Lemma 2 to $P^{\prime}$ with $t=n / 9$, either there exist $x, y \in P^{\prime}$ such that $C\left(P^{\prime}, x, y\right) \geq n / 27+2$ or there exist $x, y, z \in P^{\prime}$ such that at most $n / 9$ points of $P^{\prime}$ lie outside $\triangle x y z$. If $C\left(P^{\prime}, x, y\right) \geq$ $n / 27+2$, then Theorem 2 holds, so assume there exist three points $x, y, z \in P^{\prime}$ such that at most $n / 9$ points of $P^{\prime}$ lie outside $\Delta x y z$. Place these three points in set $S_{2}$ and place the triangle in set $T$.

We repeat this procedure $n / 9$ times, each time letting $P^{\prime}$ be the set remaining points not yet assigned to $S_{1}$ or $S_{2}$. For each $P^{\prime}$ we either find an $x, y$ which satisfies Theorem 2 , or we find three points $x, y, z \in P^{\prime}$ such that at most $n / 9$ points lie outside $\Delta x y z$. If we satisfy Theorem 2 we are done, so assume we place $n / 3$ points in $S_{2}$ forming $n / 9$ triangles in $T$. Label the set of remaining $n / 3$ points $S_{3}$. Each triangle in $T$ was chosen so that at most $n / 9$ of the remaining points lay outside the triangle. Thus, each triangle in $T$ contains at least $2 n / 9$ points from $S_{3}$.

Connect all the points in $S_{1}$ to all the points in $S_{3}$ using $n^{2} / 9$ line segments. Each triangle intersects at least $2 n^{2} / 27$ line segments. Hence there are at least $2 n^{3} / 243$ intersections between triangle edges and line segments.

If line segment $(x y)$ intersects line segment $(u v)$ and every circle containing points $x$ and $y$ contains $u$ or $v$, then we say that $(x y)$ dominates $(u v)$. By Lemma 3 , if line segments $(x y)$ and $(u v)$ intersect, then either $(x y)$ dominates $(u v)$ or $(u v)$ dominates $(x y)$.

Either line segments dominate triangle edges $n^{3} / 243$ times or triangle edges dominate line segments $n^{3} / 243$ times. Assume the $n^{2} / 9$ line segments dominate triangle edge segments $n^{3} / 243$ times. Some line segment, say $(x y)$, dominates at least $n / 27$ triangle edges. These $n / 27$ triangle edges come from $n / 27$ distinct triangles and must have distinct endpoints. Therefore, any circle containing $x$ and $y$ must contain $n / 27+2$ points of $P$. 
Now, assume the $n / 3$ triangle edges dominate line segments $n^{3} / 243$ times. Some triangle edge, say $(x y)$, dominates at least $n^{2} / 81$ line segments. Form the bipartite graph with these $n^{2} / 81$ line segments. Each vertex in this graph has maximum degree $n / 3$. By Lemma 4 there exists a matching using $n / 27$ edges. $(x y)$ dominates $n / 27$ line segments where no two line segments share an endpoint. Therefore, any circle containing $x$ and $y$ contains $n / 27+2$ points.

\section{Upper Bounds}

In this section we prove:

Theorem 3. $\bar{\Pi}(n) \leq\lceil n / 3\rceil+1$

and

Theorem 4. $\Pi(n) \leq\lceil n / 4\rceil+1$.

To prove Theorem 3 we show how to construct a convex configuration of $n$ points $K$ such that for every pair of points $u, v \in K, C(K, u, v) \leq\lceil n / 3\rceil+1$. Draw an equilateral triangle with sides of unit length in the plane and label its vertices $x, y$, and $z$. (If desired, we can replace edges $(x y),(y z)$, and $(z x)$ with arcs of large circles to ensure that no three points are collinear.) Place $\lfloor n / 3\rfloor$ points on edge $(x y)$ close to $x,\lfloor n / 3\rfloor$ points on edge $(y z)$ close to $y$, and $[n / 3\rfloor$ points on edge $(z x)$ close to $z$ (see Fig. 3). Distribute any remaining points among the three groups. The resulting set of points $K$ is convex. We leave it to the reader to show that through any two points there is a circle containing $[n / 3\rceil+1$ points.

To prove Theorem 4 we show how to construct general configurations of $n$ points $P$ so that for every pair of points $u, v \in P, C(P, u, v) \leq\lceil n / 4\rceil+1$. Again draw an equilateral triangle with sides of unit length and vertices labeled $x, y$, and $z$. Let $s$ be the midpoint of edge $\overline{y z}$ and let $r$ be the midpoint of edge $\overline{x y}$. Let $w$ be a point on line $(x s)$ one unit from $x$ and farther from $s$ than from $x$. Place $[n / 4\rfloor$ of the points on line segment $(x y),[n / 4\rfloor$ points on line segment $(y z)$, and $\lfloor n / 4\rfloor$ points on line segment $(z x)$ as before. Place $\lfloor n / 4\rfloor$ of the points on line segment ( $w r$ ) near $w$ (see Fig. 4). Distribute any remaining points among the four groups. We again leave it to the reader to show that through any two points there is a circle containing $\lceil n / 4\rceil+1$ points.

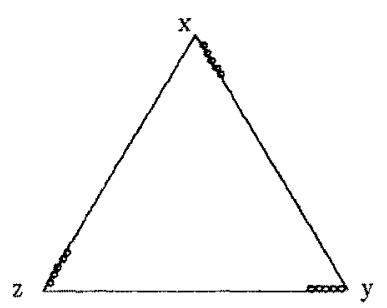

Fig. 3. Convex configuration. 


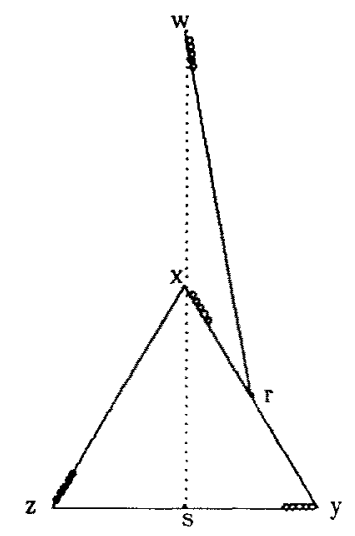

Fig. 4. General configuration.

\section{Conclusion}

We have shown that $\bar{\Pi}(n)=\lceil n / 3\rceil+1$. However, exact bounds for $\Pi(n)$ remain open. We feel that our lower bounds are still fairly loose and so we conjecture that $\Pi(n) \sim n / 4$. We are also interested in algorithms to find $x, y \in K$ such that $C(K, x, y)=\bar{\Pi}(n)$ and $x, y \in P$ such that $C(P, x, y)=\Pi(n)$ and in algorithms to find $x, y \in K$ which maximize $C(K, x, y)$ and $x, y \in P$ which maximize $C(P, x, y)$. Finally, we note that Schmerl et al. [4] have recently achieved results on the generalization of this problem to $d$-dimensional space.

\section{Acknowledgments}

We would like to thank David Avis for bringing this problem to our attention and reviewing our solutions and Vašek Chvátal for his help in some graph theoretical results used in this paper. We would also like to thank Bill Lenhart tor his corrections and suggestions.

\section{References}

1. J. A. Bondy and U. S. R. Murty, Graph Theory with Applications, 74-75, 1976.

2. V. Neumann-Lara and J. Urrutia, A Combinatorial Result on Points and Circles on the Plane, Technical Report TR-85-15, University of Ottawa, 1985.

3. J. H. Schmerl, Private communication, University of Connecticut.

4. J. H. Schmerl, S. J. Sidney and J. Urrutia, A combinatorial result about points and balls in Euclidean space, preprint.

Received August 12, 1986, and in revised form February 26, 1987. 